\title{
Critérios sociais e éticos de priorização de pacientes: uma pesquisa a estudantes e profissionais de saúde em Portugal
}

\author{
Social and ethical criteria for prioritizing patients: a survey of \\ students and health professionals in Portugal
}

Micaela Moreira Pinho ${ }^{1}$

${ }^{1}$ Departamento Economia, Gestão e Informática (DEGI), Universidade Portucalense. R. Dr. António Bernardino de Almeida 541, Polo Universitário da Asprela. 4200-072 Porto Portugal.michaelapinho@ hotmail.com

\begin{abstract}
This qualitative/quantitative study examines the ethical dilemma of microallocation of health resources. It seeks to identify and compare the opinion of two groups in Portuguese society - students and health professionals - on the importance of personal characteristics of patients at the moment of prioritizing them and if the choices can be explained by bioethical references of a utilitarian or deontological nature. Data were collected by means of a questionnaire administered to a sample of 180 students and 60 health professionals. Faced with hypothetical emergency scenarios, the respondents had to choose between two patients (distinguished by: age, gender, social responsibility, economic and employment situation, harmful health behaviors and criminal record), duly selecting who to treat and then justifying their choice. The results suggest the existence of differences in choices between the two groups, with health professionals revealing they are less prepared to accept the use of social criteria in a context of scarce resources and co-existence of utilitarian and deontological criteria, with a predominance of efficiency on the part of health professionals and equity on the part of students.
\end{abstract}

Key words Patient selection, Utilitarianism, Equity, Ethical criteria
Resumo O estudo quali-quantitativo explora o dilema ético da microalocação dos recursos da saúde. Objetiva identificar e comparar a opinião de dois grupos da sociedade portuguesa - estudantes e profissionais de saúde sobre a importância das características pessoais dos pacientes no momento de os priorizar e se as escolhas se explicam por referenciais bioéticos de caráter utilitaristas ou deontológicos. Os dados foram recolhidos através de um questionário aplicado a uma amostra de 180 estudantes universitários e 60 profissionais de saúde. Os respondentes perante hipotéticos cenários de emergência clinica tiveram de escolher de entre dois pacientes (distinguidos por idade, sexo, responsabilidade social, situação económica e laboral, comportamentos lesivos da saúde e registo criminal) quem tratar e justificar a escolha. Foram usados testes estatísticos de associação para comparar as respostas dos dois grupos e análise de conteúdo para categorizar as justificações. Os resultados sugerem a existência de diferenças nas escolhas dos dois grupos, com os profissionais de saúde a revelarem aceitar menos a utilização de critérios sociais em contexto de escassez e coexistência de critérios utilitaristas e deontológicos, com predomínio da eficiência por parte dos profissionais de saúde e da equidade por parte dos estudantes.

Palavras-chave Seleção de pacientes, Utilitarismo, Equidade, Critérios éticos 


\section{Introdução}

O direito à saúde pode estar comprometido pela insuficiência de recursos (sejam de caráter físico, tecnológico, humano e/ou financeiro) que caracteriza os sistemas de saúde públicos contemporâneos. Racionar na área da saúde é controverso e conflituoso, sobretudo ao nível micro quando se torna necessário decidir que doentes tratar. $\mathrm{O}$ desafio que se coloca aos decisores políticos, acadêmicos e profissionais de saúde é o de definir critérios que norteiem a escolha dos pacientes. Um critério amplamente difundido pelos economistas da saúde sustenta que o melhor esquema distributivo é aquele que maximiza o número de anos de vida (saudáveis) na sociedade para uma determinada disponibilidade de recursos. Este critério da maximização dos benefícios de saúde é encarado como uma regra de escolha consequencial que pouco tem a dizer quanto à multiplicidade de valores que dominam a forma como os stakeholders pensam o problema das escolhas ao nível micro. Nas decisões distributivas a evidência indica que o potencial ganho de saúde não é a única fonte de valor social. A sociedade revela igualmente preocupações equitativas traduzidas na valoração de algumas características pessoais dos doentes (Pinho e Veiga ${ }^{1}$ para uma revisão). Têm surgido na literatura inúmeras propostas de princípios distributivos que suportam as considerações éticas imiscuídas na seleção de pacientes ${ }^{2-4}$. Estes princípios podem ser enquadrados em critérios éticos, mais gerais e potencialmente conflituosos, de caráter teleológico (utilitarismo) ou deontológico (equidade). Este artigo começa por categorizar os princípios distributivos nestes dois critérios e discute alguma da evidência empírica sobre as preferências sociais nesta matéria. Segue-se a apresentação dos resultados de um estudo exploratório quali-quantitativo desenhado para obter e comparar as preferências de dois grupos da sociedade Portuguesa - estudantes e profissionais de saúde - quanto aos critérios sociais que devem sustentar a seleção dos doentes a tratar. Por fim, são discutidas as principais conclusões do estudo e apresentada a agenda de investigação futura.

\section{Critérios éticos inerentes à priorização de doentes}

A bioética tem procurado compreender os valores morais envolvidos na priorização dos doentes. Neste sentido, sobressaem duas correntes éticas - teleológicas (utilitarismo) e deontológi- cas (equidade). O utilitarismo avalia a bondade das ações de acordo com a sua finalidade, isto é, procura o máximo bem-estar (utilidade social) para o maior número de pessoas. Quando aplicado à saúde, o utilitarismo pode revelar-se numa vertente pecuniária ou social ${ }^{5}$. O utilitarismo pecuniário é popular entre os economistas da saúde que, movidos pela prossecução da eficiência, defendem a afetação dos recursos de acordo com a maximização da saúde esperada da população - efectividade clínica ${ }^{6}$. Neste contexto, recebe prioridade o paciente que apresenta melhor prognóstico. $\mathrm{O}$ caráter pecuniário do utilitarismo estende-se a considerações indiretas como seja a capacidade produtiva da pessoa tratada. Assim a prioridade conferida a indivíduos pertencentes a classes socioeconómicas mais elevadas ou empregados justifica-se pelo seu maior contributo para a criação de riqueza. O utilitarismo social incorpora externalidades ao avaliar o efeito dos tratamentos sobre terceiros. São atribuídas ponderações ao doente de acordo com o seu contributo para o bem-estar social, numa filosofia que extravasa a teoria do capital humano. Neste sentido, por garantir o máximo bem-estar para o maior número de pessoas merece prioridade o doente que tem dependentes a cargo. Estas preferências refletem que o efeito que um indivíduo exerce sobre a sua família ou amigos não é facilmente substituível, contrariamente ao seu papel contributivo?

A ética deontológica avalia a bondade das ações de acordo com o seu motivo ou intenção. A justiça é equiparada a equidade e, neste caso, uma escolha será considerada boa se estiver em conformidade com uma norma moral. Equidade em saúde é um conceito polissémico e amplamente estudado na literatura. Ainda que não exista uma teoria geral e universal do que se entende por equidade em saúde, uma ideia que se tem generalizado equipara equidade à redução das desigualdades em saúde 8 . Este tipo de preocupação pode ser considerada prospetivamente ou retrospetivamente, consoante os efeitos dos tratamentos sejam julgados em termos futuros ou, antes, em termos de ações passadas ${ }^{5}$. A teoria mais geral de justiça defendida por Rawls ${ }^{9}$ e traduzida na regra "maximin" prevê uma redução prospetiva das desigualdades ao aceitar distribuições que, mesmo podendo ser injustas, privilegiem os indivíduos mais desfavorecidos da sociedade. Quando aplicado à saúde, pode significar dar prioridade aos pacientes mais doentes (independentemente da capacidade de benefício do tratamento), mais frágeis/vulneráveis ou provenientes de classes so- 
cioeconómicas mais baixas. Outra variante prospetiva da redução das desigualdades em saúde incide na equidade intergeracional, traduzida em igualdade na saúde usufruída ao longo de toda a vida. De acordo com o critério do fair-inning ${ }^{10}$ ou igualitarismo etário ${ }^{11}$ todos os indivíduos têm direito a viver uma vida longa e saudável devendo assim ser conferida prioridade aos doentes que mais distam deste objetivo, geralmente os mais novos. Uma interpretação retrospectiva de redução das desigualdades em saúde encontra-se na teoria da igualdade de oportunidades substantivas $^{12}$. Fundamentado na abordagem das capacidades de Amartya Sen ${ }^{13}$, este princípio sustenta que, estando assegurada a igualdade de oportunidade à saúde qualquer discrepância nesta resultante da liberdade individual é aceitável. Em conformidade com este argumento merecem prioridade os doentes com menor responsabilidade sobre a causa da sua doença. Retrospectivamente, a redução das desigualdades na saúde pode ainda basear-se no merecimento social ${ }^{4}$. Determinados indivíduos podem ser considerados mais prioritários por exercem (ou terem exercido) um papel socialmente relevante (por exemplo, dadores de sangue ou veteranos de guerra). Na mesma lógica, recebem menor prioridade os doentes que pelas suas ações prejudicam (ou prejudicaram) a sociedade (por exemplo, criminosos).

Reconhecendo-se a importância das preferências sociais em relação à priorização de doentes têm surgido imensos estudos internacionais para avaliar a adesão das populações aos diferentes critérios sociais ${ }^{5,14-18}$. Os resultados revelam uma pluralidade de opiniões sociais, todas legítimas e defensáveis no campo da justiça distributiva.

Uma desvantagem da maioria destes estudos reside no facto de terem usado inquéritos com questões fechadas. As questões fechadas dificultam o conhecimento dos argumentos que estão inerentes a cada decisão. Por exemplo, a ideia de que os doentes mais jovens merecem prioridade sobre os mais velhos parece uma ideia consensual nos diversos estudos conduzidos. Porém, é difícil dizer se a prioridade conferida aos mais jovens se deve a motivações utilitaristas ou antes deontológicas. A preferência por doentes mais novos pode decorrer da: (i) sua maior capacidade de benefício - utilitarismo pecuniário, (ii) sua morte gerar muita infelicidade nos progenitores - utilitarismo social; (iii) necessidade de suporte físico e emocional - regra "maximin" e/ou (iv) vida que ainda têm pela frente - fair-inning. Dificuldades de interpretação similares podem ocorrer com outras características pessoais. Identificar, claramente, as razões para priorizar um doente em relação a outro é importante para averiguar se uma sociedade é, tendencialmente, movida por considerações de eficiência ou de equidade. Poucos foram, até o momento, os estudos que procuraram compreender estas motivações. Destacam-se dois ensaios conduzidos no Brasil que, através de dados qualitativos, avaliaram a adesão aos critérios da idade, gênero, responsabilidade social e estilos de vida de uma amostra de conveniência da população, em geral ${ }^{14}$ e de estudantes de pós-graduação em saúde pública. ${ }^{16}$ Os autores concluíram pela coexistência de elementos identificadores de equidade e eficiência. Enquanto a população, em geral, demonstrou uma expressiva opção pelos mais desfavorecidos da sociedade ${ }^{14}$ os estudantes pós-graduados denotaram uma clara tendência para as justificativas baseadas na ética utilitarista na vertente pecuniária e social ${ }^{16}$.

Uma segunda lacuna que pode ser apontada aos estudos empíricos conduzidos prende-se com os stakeholders envolvidos. Contrariamente à opinião da população sobre a aceitação das características pessoais dos doentes enquanto métodos de priorização estar amplamente difundida, pouco se sabe das preferências dos profissionais de saúde e, ainda menos, sobre a comparabilidade de opiniões entre estes dois grupos. Será que a população em geral e os profissionais de saúde partilham os mesmos valores morais em termos de microalocação dos recursos? Esta questão é pertinente na medida em que este tipo de escolhas cabe, em última instância, aos profissionais de saúde. Neste contexto, parece-nos importante explorar se estes dois grupos de atores sociais comungam dos mesmos critérios éticos de priorização de doentes sob pena de qualquer processo de reforma do método discricionário atualmente vigente ficar desde logo comprometido. Os poucos estudos conhecidos que compararam a opinião da população e dos profissionais de saúde revelam alguma variância entre os dois grupos $^{19-21}$. Por exemplo, Rynnanen et al. ${ }^{20}$ concluíram que a idade é um indicador de priorização mais valorado pelos médicos e enfermeiros que pela população em geral. Neuberger et al. ${ }^{19} \mathrm{e}$ Oddsson $^{21}$ concluíram que os médicos são mais adeptos do critérios da efectividade clínica que o público em geral. Salvo uma exeção ${ }^{20}$, a maioria destes estudos só avaliaram a opinião dos médicos. Uma vez que as decisões mais dramáticas de microalocação de recursos acontecem, geralmente, em contexto de urgências hospitalares, onde intervêm para além de médicos outros profissio- 
nais de saúde, como enfermeiros e técnicos, parece-nos conveniente conhecer a opinião de todos os potenciais profissionais de saúde envolvidos. Adicionalmente, incorporar a visão da população e dos profissionais de saúde, enquanto elementos no processo de tomada de decisões pode ajudar os decisores políticos a formular critérios sistemáticos, assegurando que as decisões tomadas são, se não as ideais pelo menos as legitimadas pelos principais atores sociais no processo.

\section{Metodologia}

O presente artigo procura resolver as desvantagens apontadas anteriormente à evidência empírica. Neste sentido, desenvolvemos um estudo exploratório com dois objetivos principais: Em primeiro lugar, procuramos comparar a opinião de dois grupos da sociedade portuguesa - população em geral e profissionais de saúde sobre a importância conferida a determinadas características pessoais dos doentes no momento de selecionar quem tratar. Em segundo lugar, procuramos explorar as motivações associadas a cada uma destas características no sentido de averiguar se os grupos são movidos por preocupações éticas, teleológicas ou deontológicas.

\section{Questionário}

Recorremos a uma combinação de dados quantitativos e qualitativos para identificar as características pessoais dos pacientes mais valoradas quando, na decorrência da escassez de recursos, é necessário limitar o acesso aos cuidados de saúde. Os dados foram recolhidos através de um questionário individualizado composto por duas partes. Para além da caracterização sociodemográfica dos respondentes, o questionário apresentava 10 cenários hipotéticos de emergência clínica, a maioria dos quais previamente desenhados e testados ${ }^{14,16}$. Em cada cenário, os respondentes foram confrontados com a escolha entre dois pacientes chegados a um serviço de urgência, que só dispõe de recursos para tratar um deles. Ambos os pacientes apresentavam o mesmo risco de vida, se não tratados. Os pacientes distinguiam-se apenas pelas suas características pessoais, que agrupamos em três tipologias: (i) inerentes ao próprio indivíduo - idade e gênero; (ii) relacionadas com terceiros - responsabilidade social (estado civil e dependentes), estatuto socioeconómico e situação profissional e (iii) inerentes a comportamentos de saúde e sociais
- fumar, beber, registo criminal. Foi solicitado a cada respondente que justificasse, num espaço para o efeito, convenientemente a sua escolha. Não foi conferida aos respondentes a possibilidade de "não escolherem nenhum dos doentes" nem a possibilidade de darem "igual prioridade"; antes, pelo contrário, procuramos forçar os inquiridos a tomar uma decisão. Deste modo, tentamos acautelar que os inquiridos evitassem fazer escolhas e, por conseguinte, evitassem alguma desutilidade que isso pudesse gerar ${ }^{22}$. Ainda assim, no espaço deixado para a justificação da escolha, os respondentes poderiam manifestar estas preferências expondo a razão para tal. Estamos conscientes que o caráter não real dos nossos cenários pode comprometer a correta eliciação das preferências. Ainda assim, acreditamos ser possível comparar o padrão de preferências em Portugal com os demais estudos onde estas situações também não são, de todo, reais.

\section{Amostra}

O questionário foi aplicado durante o ano de 2014 a uma amostra de conveniência constituída por 180 estudantes universitários e 60 profissionais de saúde da zona norte de Portugal continental. Os respondentes foram informados sobre o caráter da pesquisa, seus objetivos, os procedimentos a serem observados, o aspecto voluntário da participação e a garantia do anonimato. A seleção das amostras foi feita em sala de aula de três universidades e em dois hospitais públicos, respetivamente, para o caso dos estudantes e profissionais de saúde. Depois de exposto o âmbito da pesquisa foi solicitada a colaboração (voluntária) dos respetivos estudantes e profissionais de saúde no preenchimento do questionário.

Os estudantes $(75 \%)$ frequentavam o último ano de licenciatura de diferentes cursos: economia (18.1\%), gestão (12.0\%), psicologia (17.5\%), direito (15\%) e medicina (12.5\%). O grupo dos profissionais de saúde (25\%) era constituído por médicos (10.8\%), enfermeiros $(9.3 \%)$ e técnicos de saúde (4.9\%). Todos os profissionais de saúde apresentavam experiência em contexto hospitalar.

No caso dos estudantes o inquérito foi preenchido em sala de aula de forma individual sem direito a discussão. Os profissionais responderam ao questionário também de forma individualizada no final do turno de trabalho. Foi concedido tempo suficiente para os respondentes refletirem no tema e formularem opiniões reflexivas em linha com o defendido por Dolan et al..$^{23}$ Foi cuidadosamente explicado a todos os respondentes 
que, não obstante os cenários de racionamento apresentados serem irreais, o propósito do presente exercício é o de definir os princípios éticos gerais que suportam.

A amostra final dos estudantes era composta por $52.4 \%$ de mulheres. Os estudantes tinham idades compreendidas entre os 18 e os 55 anos (média 24 anos), eram majoritariamente (68\%) solteiros e o rendimento líquido mensal mediano do agregado familiar variava entre $800 €$ e $1500 €$. A amostra final dos profissionais de saúde era, majoritariamente, do sexo feminino (60\%) e pertenciam à faixa etária 24-65 anos (média 40 anos). Os profissionais eram na sua maioria casados $(56.7 \%)$ e dispunham de um rendimento mensal líquido, mediano, entre $800 €$ e $2500 €$.

\section{Análises}

O tratamento quantitativo dos dados foi prosseguido com auxílio do programa SPSS (versão 20). Foram conduzidos testes estatísticos de associação (Qui-quadrado) a um nível de significância de 5\%. A influência das características demográficas dos respondentes na distribuição das suas respostas foi testada a partir de análises bivariadas e multivariadas. Os dados qualitativos foram tratados através da análise de conteúdo ${ }^{24}$. Recorremos à análise de conteúdo para sumariar e sistematizar as justificativas avançadas pelos respondentes para a escolha entre os pacientes. Os temas relevantes foram identificados e categorizados em categorias e subcategorias. A abordagem consistiu em codificar grupos de palavras usando um esquema de classificação construído através de um processo interativo. O processo interativo de categorização classificou as ideias em seis categorias gerais correspondentes aos princípios de racionamento identificados na literatura (e descritos anteriormente). As razões referenciadas pelos respondentes foram assim codificadas em "Princípios" (categoria principal) e "Argumentos" (subcategoria). Os princípios correspondem aos critérios gerais de distribuição de cuidados de saúde e os argumentos retratam aspetos específicos da tomada de decisões e foram subcategorizadas nas várias variantes de cada princípio. Cada princípio foi, por sua vez, enquadrado no correspondente critério ético teleológico ou deontológico.

\section{Resultados}

\section{Análise Quantitativa}

A Tabela 1 sintetiza a distribuição das respostas de cada um dos grupos de inquiridos - estudantes e profissionais de saúde, a cada um dos cenários. Para além da ocupação dos respondentes nenhuma outra variável demográfica se revelou estatisticamente significativa na distribuição das respostas. A primeira coluna da Tabela 1 apresenta os diversos cenários hipotéticos e os correspondentes critérios de escolha envolvidos. A terceira e quarta coluna sintetizam a distribuição das escolhas dos estudantes e dos profissionais de saúde, respetivamente, para cada cenário. A opção que se apresenta como "não escolha” resulta da resposta dada pelos inquiridos no espaço deixado para a justificação. Codificamos como "não escolha" todas as respostas dos inquiridos que se recusaram a escolher um doente ou que lhes conferiram, expressamente, igual prioridade. A última coluna apresenta o valor da estatística do teste Qui-Quadrado. As diferenças com significância estatística estão assinaladas com um asterisco.

Os resultados revelam, de forma geral, a existência de diferenças estatisticamente significativas entre estudantes e profissionais de saúde quanto à importância atribuída às características pessoais dos pacientes no momento de os priorizar. Excetuam-se, o fator idade e os comportamentos lesivos da saúde. Ambos os grupos priorizam a criança face ao idoso $(>90 \%)$ ou ao adulto $(>80 \%)$. Os fumadores e alcoólicos são preteridos por ambos os grupos, notando-se uma maior penalização do álcool do que do tabaco. Nos restantes cenários é possível concluir que os profissionais de saúde conferem, majoritariamente, igual prioridade a ambos os pacientes. As diferenças quantitativas são mais notórias no fator gênero (83.3\% contra $23.9 \%$ de estudantes), situação profissional (50\% contra $24,4 \%$ de estudantes), estatuto socioeconómico (58.3\% contra $27.8 \%$ de estudantes) e registo criminal $(80 \%$ contra $24.49 \%$ de estudantes). A diferença entre os dois grupos de inquiridos é menos acentuada quando está em causa a responsabilidade social do doente (estado civil e dependentes). Metade dos profissionais de saúde revelou preferir a mulher casada (contra 94.4\% dos estudantes), ainda que $43.3 \%$ não tenha condicionado o acesso ao tratamento pelo estado civil. A mulher com filhos foi considerada prioritária por $80 \%$ dos profissionais de saúde e $94.4 \%$ dos estudantes. Ainda 
Tabela 1. Percentagem respostas a cada cenário por estudantes e profissionais de Saúde.

\begin{tabular}{|c|c|c|c|c|}
\hline \multirow{2}{*}{$\begin{array}{c}\text { Cenários } \\
\text { Num serviço de urgência chegam dois doentes em } \\
\text { perigo de vida mas só existem recursos para atender } \\
\text { um. Quem escolher entre ... }\end{array}$} & \multirow[b]{2}{*}{ Opções } & \multicolumn{2}{|c|}{ Resultados } & \multirow[b]{2}{*}{$\chi^{2}$} \\
\hline & & $\begin{array}{c}\text { Estudantes } \\
(\%)\end{array}$ & $\begin{array}{l}\text { Profissionais } \\
\text { Saúde (\%) }\end{array}$ & \\
\hline \multirow{3}{*}{$\begin{array}{l}\text { 1. Criança } 8 \text { anos \& Idoso } 60 \text { anos } \\
\text { (Critério: Idade) }\end{array}$} & Criança & 92.2 & 91.7 & \multirow[t]{3}{*}{7.816} \\
\hline & Idoso & 5.6 & 0.0 & \\
\hline & Não escolhe & 2.2 & 8.3 & \\
\hline \multirow{3}{*}{$\begin{array}{l}\text { 2. Criança } 10 \text { anos \& Adulto } 40 \text { anos } \\
\text { (Critério: Idade) }\end{array}$} & Criança & 83.3 & 80.0 & \multirow[t]{3}{*}{9.697} \\
\hline & Adulto & 11.1 & 3.3 & \\
\hline & Não escolhe & 5.6 & 16.7 & \\
\hline \multirow{3}{*}{$\begin{array}{l}\text { 3. Homem \& Mulher } \\
\text { (Critério: Género) }\end{array}$} & Mulher & 68.3 & 15.0 & \multirow[t]{3}{*}{$66.997^{*}$} \\
\hline & Homem & 7.8 & 1.7 & \\
\hline & Não escolhe & 23.9 & 83.3 & \\
\hline \multirow{3}{*}{$\begin{array}{l}\text { 4. Mulher casada \& solteira } \\
\text { (Critério: Responsabilidade Social) }\end{array}$} & Casada & 73.3 & 50.0 & \multirow[t]{3}{*}{$17.889^{*}$} \\
\hline & Solteira & 10.0 & 6.7 & \\
\hline & Não escolhe & 16.7 & 43.3 & \\
\hline \multirow{3}{*}{$\begin{array}{l}\text { 5. Mulher com \& sem filhos } \\
\text { (Critério: Responsabilidade Social) }\end{array}$} & Com filhos & 94.4 & 80.0 & \multirow[t]{3}{*}{$21.543^{*}$} \\
\hline & Sem filhos & 2.8 & 0.0 & \\
\hline & Não escolhe & 2.8 & 20.0 & \\
\hline \multirow{3}{*}{$\begin{array}{l}\text { 6. Fumador \& não Fumador } \\
\text { (Critério: Comportamentos Risco) }\end{array}$} & Fumador & 21.1 & 21.7 & \multirow[t]{3}{*}{5.70} \\
\hline & Não Fumador & 75.6 & 66.7 & \\
\hline & Não escolhe & 3.3 & 11.7 & \\
\hline \multirow{3}{*}{$\begin{array}{l}\text { 7. Alcoólico \& Não alcoólico } \\
\text { (Critério: Comportamentos Risco) }\end{array}$} & Alcoólico & 18.3 & 11.7 & \multirow[t]{3}{*}{1.758} \\
\hline & Não Alcoólico & 77.2 & 81.7 & \\
\hline & Não escolhe & 4.4 & 6.7 & \\
\hline \multirow{3}{*}{$\begin{array}{l}\text { 8. Empregado \& Desempregado } \\
\text { (Critério: Situação Laboral) }\end{array}$} & Empregado & 53.9 & 36.7 & \multirow[t]{3}{*}{$13.819^{*}$} \\
\hline & Desempregado & 21.7 & 13.3 & \\
\hline & Não escolhe & 24.4 & 50.0 & \\
\hline \multirow{3}{*}{$\begin{array}{l}\text { 9. Elevado \& Baixo Rendimento } \\
\text { (Critério: Estatuto socioeconómico) }\end{array}$} & Elevado Rend. & 19.4 & 8.3 & \multirow[t]{3}{*}{$18.747^{*}$} \\
\hline & Baixo Rend. & 52.8 & 33.3 & \\
\hline & Não escolhe & 27.8 & 58.3 & \\
\hline \multirow{3}{*}{$\begin{array}{l}\text { 10. Ex Presidiário \& Não cadastrado } \\
\text { (Critério: Merecimento) }\end{array}$} & Ex Presidiário & 8.9 & 0.0 & \multirow[t]{3}{*}{$59.383^{*}$} \\
\hline & Não Cadastrado & 66.7 & 20.0 & \\
\hline & Não escolhe & 24.4 & 80.0 & \\
\hline
\end{tabular}

*Nível de significância $\mathrm{p}<0.001$.

assim, 20\% dos profissionais de saúde não valorizou a dependência dos filhos (contra apenas $2.8 \%$ dos estudantes).

\section{Análise Qualitativa}

A apresentação dos resultados qualitativos versou sobre os princípios (categorias principais) e os argumentos (subcategorias) identificados no questionário, tanto por grupo de respondentes como entre os grupos (estudantes e profissionais de saúde). O Quadro 1 apresenta os princípios e argumentos por cada critério ético por grupo de respondentes. Todas as respostas dadas pelos respondentes estão contempladas no Quadro 1. O levantamento das justificativas invocadas pelos respondentes para as suas escolhas revela que estas se enquadram dentro dos princípios distributivos propostos na literatura. Segue-se uma descrição detalhada das razões avançadas por cada grupo de inquiridos.

\section{Estudantes}

Os estudantes revelam, simultaneamente, preocupações utilitaristas e de equidade. As motivações utilitaristas de cariz pecuniário estão presentes nos argumentos avançados a favor das crianças, dos adultos, dos empregados e dos não fumadores/alcoólicos. Foi frequente a alusão à maior capacidade de benefício das crianças e ao seu futuro papel contributivo. O papel con- 
Quadro 1. Razões invocadas por estudantes e profissionais de Saúde.

\begin{tabular}{|c|c|c|c|c|c|}
\hline & \multicolumn{2}{|c|}{ Princípios Éticos } & Argumentos & Estudantes & $\begin{array}{c}\text { Profissionais } \\
\text { Saúde }\end{array}$ \\
\hline & & & $\begin{array}{l}\text { "Não cabe a ninguém tomar estas decisões" } \\
\text { "os doentes são todos iguais" } \\
\text { "selecionar pacientes com base, apenas, } \\
\text { nestas características não faz sentido" }\end{array}$ & $\begin{array}{l}\checkmark \\
\checkmark \\
--\end{array}$ & 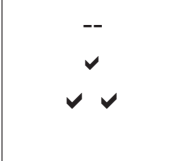 \\
\hline \multirow[t]{2}{*}{ 量 } & \multicolumn{2}{|l|}{ Pecuniário } & $\begin{array}{l}\text { "criança maior capacidade recuperação" } \\
\text { "criança será futuro contribuinte" } \\
\text { "adulto é um contribuinte" } \\
\text { "criança beneficia do tratamento mais } \\
\text { tempo" } \\
\text { "empregados estão a contribuir para a } \\
\text { sociedade" } \\
\text { "fumadores/alcoólicos têm menor } \\
\text { capacidade recuperação" }\end{array}$ & $\begin{array}{l}\checkmark \\
\checkmark \\
\checkmark \\
\checkmark \\
\checkmark\end{array}$ & $\begin{array}{c}\checkmark \checkmark \\
-- \\
-- \\
\checkmark \\
-- \\
\checkmark\end{array}$ \\
\hline & \multicolumn{2}{|l|}{ Social } & $\begin{array}{l}\text { "mulher pode ter filhos" } \\
\text { "mulher tem a família para cuidar" } \\
\text { "a morte da criança provoca uma dor } \\
\text { intolerável aos pais" }\end{array}$ & $\checkmark$ & $\begin{array}{l}-- \\
- \\
-\end{array}$ \\
\hline \multirow{4}{*}{ 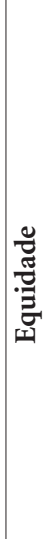 } & \multirow[t]{2}{*}{ Prospetiva } & "Maximin" & $\begin{array}{l}\text { "mulheres são mais frágeis" } \\
\text { "crianças são mais frágeis/vulneráveis" } \\
\text { "idosos são mais frágeis/vulneráveis" } \\
\text { "desempregados/pobres não podem recorrer } \\
\text { a saúde privada" }\end{array}$ & $\begin{array}{l}\grave{v} \\
\checkmark \\
\checkmark\end{array}$ & $\begin{array}{l}-- \\
-- \\
--\end{array}$ \\
\hline & & Fair-Inning & "criança tem uma vida pela frente" & $\checkmark \checkmark$ & $\checkmark$ \\
\hline & \multirow[t]{2}{*}{ Retrospetiva } & $\begin{array}{l}\text { Igualdade } \\
\text { Oportunidades } \\
\text { Substantivas }\end{array}$ & $\begin{array}{l}\text { "fumadores e/ou alcoólicos sabem que isso } \\
\text { prejudica a saúde" }\end{array}$ & $\checkmark$ & -- \\
\hline & & Merecimento & $\begin{array}{l}\text { "validade social do indivíduo" } \\
\text { "maior contributo social do não cadastrado" }\end{array}$ & $\checkmark$ & $\begin{array}{l}-- \\
--\end{array}$ \\
\hline
\end{tabular}

$\checkmark=$ mencionado; $\checkmark \checkmark=$ mencionado com muita frequência; -- não mencionado.

tributivo foi, igualmente, a justificação avançada por aqueles que deram prioridade ao adulto em detrimento da criança e ao empregado em detrimento do desempregado. Este argumento produtivo em favor do adulto e do empregado encontra uma aplicação prática no cálculo dos disability-adjusted life years (DALYs) ou burden of disease $e^{25}$. De forma similar, os atos de fumar e beber foram reconhecidos, pelos respondentes, como condicionantes da capacidade de beneficiar do tratamento. O utilitarismo de caráter social foi evidenciado pela prioridade conferida em função do gênero. $\mathrm{O}$ homem foi majoritaria- mente preterido pelos estudantes que encaram a mulher como o suporte emocional da família e essencial para a continuidade da espécie humana.

As justificativas avançadas pelos estudantes revelam, ainda, fortes preocupações equitativas. A ética deontológica foi evidenciada na prioridade conferida com base na fragilidade/vulnerabilidade do doente tanto em termos físicos (criança, idosos, mulheres) como financeiros (pobres, desempregados). A preferência pela mulher neste contexto não deixa de ser algo surpreendente. Apenas poderemos especular quanto à razão desta preferência pela mulher. Pode, eventualmen- 
te, existir um efeito proximidade. O facto de os estudantes serem muito jovens pode justificar que se sintam emocionalmente muito ligados à figura materna. Ainda no campo da equidade prospetiva, foi generalizada a defesa da igualdade inter-generacional com a prioridade conferida aos mais novos. Em termos retrospetivos penalizaram-se os comportamentos, socialmente reconhecidos como lesivos da saúde. Esta tendência foi reforçada com base na validade social dos doentes, traduzida na discriminação negativa do ex -presidiário. Por fim, importa referir que apenas uma minoria dos inquiridos revelou desconforto com este tipo de exercício catalogando esta forma de decisões como imoral. Neste sentido, recusaram selecionar qualquer doente alegando que todos são igualmente merecedores de cuidados de saúde. Esta justificativa encontra suporte teórico no princípio da loteria ${ }^{26}$.

\section{Profissionais de Saúde}

Os profissionais de saúde denotam, à semelhança dos estudantes, preocupações utilitaristas e equitativas. Ainda assim, os profissionais de saúde preocupam-se essencialmente com a efectividade clínica, facto que os leva a priorizar a criança relativamente aos adultos e/ou idosos. A capacidade de benefício, um melhor prognóstico e uma maior resiliência são critérios que entendem essenciais e acima de todos os outros para conferir prioridade a um doente. A mesma razão foi apontada para discriminar contra os fumadores e alcoólicos. Uma forma de utilitarismo social foi proferida por aqueles que entendem que a mulher com filhos deve ter prioridade. Ainda assim, esta justificação foi avançada sobretudo por enfermeiros e técnicos de saúde. Motivações equitativas foram também manifestadas, ainda que, de forma menos intensa, com base no argumento do fair-inning. De entre os profissionais de saúde, os médicos foram os menos adeptos deste argumento. Para eles, a prioridade conferida à criança justifica-se, apenas, por considerações de efetividade clínica. Por último, a maioria dos profissionais de saúde entende que estabelecer prioridades com base, unicamente, nestas características não faz sentido recusando fazer qualquer escolha.

\section{Discussão}

O presente estudo foi desenhado para conhecer as preferências sociais pela microalocação dos escassos recursos da saúde. Recorrendo a uma me- todologia mista procuramos explorar a opinião dos estudantes e dos profissionais de saúde pelos critérios sociais e respectivas motivações éticas, que devem definir as prioridades entre doentes. Os nossos resultados indicam que os dois grupos não parecem partilhar os mesmos valores sociais. Em termos gerais, discriminar o acesso aos serviços de saúde a partir das características pessoais dos doentes parece melhor aceite pelos estudantes que pelos profissionais de saúde. Contrariamente aos profissionais de saúde, a generalidade dos estudantes não se recusou, em nenhum cenário, a escolher o doente a tratar. Os profissionais de saúde, por outro lado, revelam uma tendência mais igualitária conferindo a mesma prioridade aos doentes. Ainda assim, a opinião dos dois grupos parece convergir no fator idade, nos estilos de vida e, de forma menos marcada, na responsabilidade social. Contudo, os dois grupos são movidos por motivações éticas distintas. Embora os resultados qualitativos revelem a coexistência de preocupações utilitaristas e de equidade por parte de ambos os grupos inquiridos, os profissionais de saúde aderem, sobretudo, ao critério utilitarista pecuniário ao definirem a efetividade clínica do tratamento como indicador de prioridade. Esta ideia converge com alguns resultados internacionais ${ }^{27-30}$. Os estudantes, ainda que valorem o potencial ganho de saúde, revelam-se, igualmente, preocupados com o bem-estar de outros indivíduos relacionados com o doente. Isto encontra expressão na prioridade conferida à mulher casada e com filhos. Os estudantes revelaram, adicionalmente, alguma aversão pela desigualdade na saúde. Neste contexto, denota-se um pluralismo de valores morais, predominando a preocupação com os mais vulneráveis da sociedade (principio "maximin") e com a igualdade na esperança de vida (princípio fair-inning). A equidade intergeneracional revelou-se também uma preocupação dos profissionais de saúde, sendo, porém, menos valorada pelos médicos. Estes também foram, dentre os profissionais de saúde, os que menos valoraram a responsabilidade social. Valorar o critério do merecimento parece ser outro ponto de discordância entre os dois grupos. Contrariamente aos estudantes, os profissionais de saúde não parecem aceitar limitar o direito a cuidados de saúde ao passado criminal do paciente. Este resultado reforça a falta de consenso que existe na literatura quanto à validade do merecimento enquanto critérios social diferenciador dos pacientes. Se existe evidência de que há sociedades que aceitam esta prática ${ }^{19}$ também existe evidência em sentido oposto ${ }^{31}$. 
Por fim, seria interessante comparar os nossos resultados com os internacionais. Contudo, a diversidade de amostras e desenhos de estudos usados não facilitam a comparabilidade entre os estudos nem, tampouco, permite a formulação de conclusões detalhadas. Ainda assim, vale a pena confrontar os resultados deste estudo com os encontrados noutros trabalhos conduzidos no Brasil por terem usado uma metodologia idênti$\mathrm{ca}$, ainda que com diferentes amostras. O padrão de preferências dos nossos estudantes inquiridos parece convergir para os resultados obtidos pela amostra da população no Brasil ${ }^{14}$. Ambos denotam preocupações, tendencialmente, equitativas em favor dos mais frágeis e vulneráveis. São, porém, notórias as diferenças no padrão de respostas entre os nossos profissionais de saúde inquiridos e os do estudo conduzido no Brasil ${ }^{16}$. Ainda que ambos valorem o fator idade (conferindo prioridade ao doente mais jovem), os inquiridos no Brasil valorizam igualmente outros fatores, como o gênero e o estado civil, contrariamente ao que é revelado no nosso estudo. Uma razão para esta diferença pode estar no fato de apenas $37.5 \%$ dos profissionais de saúde inquiridos no Brasil ter experiência hospitalar. Esta justificação é, porém, meramente especulativa.

\section{Conclusões}

A insuficiência de recursos na saúde, agravada, em Portugal, por duas décadas de estagnação e retração econômica, torna urgente a formulação de orientações e critérios definidores das procuras a atender. Independentemente da maior ou menor concordância com os critérios sociais na seleção de pacientes a tratar ${ }^{22,32,33}$ decisões de priorização têm de ser tomadas. Estas decisões são inevitavelmente controversas, por privilegiarem umas necessidades sobre outras. Discute-se cada vez mais a urgência de converter a microalocação dos recursos num processo explícito, em que os critérios e as razões que ditam as decisões sejam claramente definidos. Esta pertinência começa a revelar-se por parte dos próprios profissionais de saúde, responsáveis em última instância pela seleção dos doentes a tratar. À medida que a escassez de recursos se vai acentuando, os profissionais de saúde, sobretudo os médicos, ficam cada vez mais expostos aos juízos da opinião pública. Neste sentido, acreditamos que num futuro próximo os profissionais de saúde desejem a partilha dessa responsabilidade com os políticos e admi- nistradores/gestores hospitalares, que, através das suas decisões a nível macro, dimensionam os recursos que devem distribuir de modo racional e eficiente.

Num primeiro esforço para avaliar a pertinência desta matéria prosseguimos um estudo exploratório com o intuito de avaliar se a opinião dos estudantes universitários sobre os critérios sociais que devem determinar as prioridades entre doentes e as suas razões éticas convergem com a dos profissionais de saúde. Concluímos, pela existência de diferenças de opiniões entre os dois grupos. Os estudantes mostram-se mais receptivos que os profissionais de saúde à ideia de estabelecer prioridades entre doentes com base nas suas características pessoais. Os estudantes defendem um pluralismo de valores morais com suporte pela ética teleológica em ambas as versões (pecuniária e social), mas com uma tendência clara em favor da corrente deontológica em geral e do princípio "maximin" e fair-inning, em particular. Os profissionais de saúde parecem suportar, essencialmente, o princípio utilitarista pecuniário, privilegiando o valor terapêutico dos tratamentos.

Este potencial conflito de opiniões pode obstaculizar qualquer tentativa de dotar a microalocação dos recursos de maior transparência. Os nossos resultados apontam para a necessidade de promover uma discussão pública e esclarecedora sobre estas matérias e, uma vez que as pessoas, em geral, parecem não se demitir de fazer escolhas, conhecer melhor as suas atitudes e preocupações para alcançar um acordo entre cidadãos e profissionais de saúde sobre quem tratar quando a realidade inviabiliza o atendimento de todas as necessidades.

Os resultados aqui apresentados devem ser analisados com cuidado devido ao caráter exploratório do estudo e às limitações que apresenta, a maior das quais é a não representatividade das amostras. Ainda assim, cremos ter conseguido os propósitos estipulados, nomeadamente, inserir Portugal nesta temática averiguando e comparando os pareceres de dois grupos da sociedade sobre os critérios e motivações morais que devem nortear a microalocação dos recursos.

Em termos de investigação futura, parece-nos que seria interessante alargar este estudo a uma amostra representativa da população portuguesa e a uma amostra representativa de profissionais de saúde em exercício efetivo de urgência clínica. Adicionalmente, seria interessante replicar este estudo para incluir a opinião dos pacientes. 


\section{Referências}

1. Pinho MM, Veiga PACV. Avaliação de Custo Utilidade como mecanismo de alocação de recursos em saúde: Revisão do Debate. Cad Saude Publica 2009; 25(2):239250.

2. Williams A, Cookson R. Equity in health. In: Culyer A, Newhouse P, editors. Handbook of Health Economics. North-Holland: Elsevier; 2000. p. 1863-1907.

3. Persad G, Wertheimer A, Emanuel E. Principles for allocation of scarce medical interventions. Lancet 2009; 329(9661):224-227.

4. Clark S, Weale A. Social Values in health priority setting: a conceptual framework. J Health Organ Manag 2012; 26(3):293-316.

5. Olsen J, Richardson J, Dolan P, Menzel P. The moral relevance of personal characteristics in setting health care priorities. Soc Sci Med 2003; 57(7):1163-1172.

6. Culyer AJ. The principle objective of the NHS should be to maximise aggregate health. In: New B, editors. Rationing: talk and action in health care. London: King's Fund and British Medical Journal Publishing Group; 1997. p. 95-100.

7. Dolan P, Olsen J. Distributing health care: economic and ethical issues. Oxford: Oxford University Press; 2002.

8. Wagstaff A. A QALY and the equity-efficiency tradeoff. J Health Econ 1991; 10(1):21-41.

9. Rawls J. A Theory of Justice. Cambridge: Harvard University Press; 1971

10. Williams A. Intergenerational equity: An exploration of the "Fair Innings" argument. Health Econ 1997; 6(2):117-132.

11. Tsuchiya A. Age-related preferences and age weighting health benefits. Soc Sci Med 1999; 48(2):267-276.

12. LeGrand J. Equity and Choice. An Essay in Economics and Applied Philosophy. London: Routledge, Taylor \& Francis Group; 1991.

13. Sen A. Equality of what? In: McMurrin S, editors. The Tanner Lectures on Human Values. Cambridge: Cambridge University Press; 1980.

14. Fortes P, Zoboli E. A study on the ethics of microallocation of scarce resources in health care. J Med Ethics 2002; 28(4):266-269.

15. Dolan P, Shaw R, Tsuchiya A, Williams A. QALY maximization and people's preferences: a methodological review of the literature. Health Econ 2005; 14(2):197208.

16. Fortes P, Pereira P. Priorização de pacientes em emergências médicas: uma análise ética. Rev Assoc Med Bras 2012; 58(3):335-340.

17. Diederich A, Salzmann D. Public preferences regarding therapeutic benefit, costs of a medical treatment and evidence-based medicine as prioritization criteria. J Public Health 2015; 23(3):137-148.

18. Exel J, Baker R, Mason H, Donaldson C, Brouwer W Public views on principles for health care priority setting: Findings of a European cross-country study using Q methodology. Soc Sci Med 2015, 126:128-137.

19. Neuberger J, Adams D, MacMaster P, Maidment A Speed M. Assessing priorities for allocation of donor liver grafts: survey of public and clinicians. BMJ 1998; 317:172-175
20. Ryynänen OP, Myllykangas M, Kinnunen J, Ta- kala J Attitudes to health care prioritisation meth- ods and criteria among nurses, doctors, politicians and the general public. Soc Sci Med 1999; 49(11):1529-1539.

21. Oddsson K. Assessing attitude towards prioritizing in healthcare in Iceland. Health Policy 2003; 66(2):135146.

22. Coast J. Explicit rationing, deprivation, disutility and denial disutility: evidence from a qualitative study. In: Coulter A, Ham C, editors. The Global Challenge of Health Care Rationing. New York: Open University Press; 2000.

23. Dolan P, Cookson R, Ferguson B. Effect of discussion and deliberation on the public's views of priority setting in health care: Focus group study. BMJ 1999; 318(7188):916-919.

24. Bardin L. Análise de conteúdo. Lisboa: Edições 70; 2013

25. Murray C. Quantifying the burden of disease: the technical basis for disability-adjusted life years. Bull World Health Organ 1994; 72(3):429-445.

26. Childress J. Who Shall Live When Not All Can Live. Soundings 1970; 53:339-355. Reprinted: Intervention and Reflection: Basic Issues in Ronald Munson Belmont California. Medical Ethics. Wadsworth Publishing Company. 1983: 497-505.

27. Ginsburg ME. A survey of physician attitudes and practices concerning cost-effectiveness in patient care. West J Med 2000; 173(6):390-394.

28. Hurst S, Slowther A-M, Forde R, Pegoraro R, Reiter-Theil S, Perrier A, Garrett-Mayer E, Danis M. Prevalence and Determinants of Physician Bedside Rationing. Data from Europe. J Gen Intern Med 2006, 21(11):1138-1143.

29. Skirbekk H, Nortvedt P. Making a difference: a qualitative study on care and priority setting in health care. Health Care Anal 2011; 19(1):77-88.

30. Winkelhage J, Schreier M, Diederich A. Priority setting in health care: Attitudes of physicians and patients. Health 2013; 5(4):712-719.

31. Culyer A. Equity - some theory and its policy implications. J Med Ethics 2001; 27(4):275-283.

32. British Medical Association. Medical ethics today: its practice and philosophy. BMJ; 1997; 299:316.

33. Beauchamp T, Childress J. Principles of Medical Ethics. New York Oxford: University Press; 2001.

Artigo apresentado em 11/07/2015

Aprovado em 15/09/2015

Versão final apresentada em 17/09/2015 